\title{
Massively-Parallelized, Deterministic Mechanoporation for Intracellular Delivery
}

Harish G. Dixit, Renate Starr, Morgan L. Dundon, Pranee I. Pairs, Xin Yang, Yanyan Zhang, Daniel Nampe, Christopher B. Ballas, Hideaki Tsutsui, Stephen J. Forman, Christine E. Brown, Masaru P. Rao*

Dr. H. G. Dixit, ${ }^{1}$ M. L. Dundon, ${ }^{2}$ P. I. Pairs, ${ }^{2}$ Dr. Y. Zhang, ${ }^{3}$ Dr. D. Nampe, ${ }^{1,3}$ Dr. H. Tsutsui, ${ }^{1,3,4}$ Dr. M. P. Rao ${ }^{1,2,3}$

\author{
${ }^{1}$ Department of Bioengineering \\ ${ }^{2}$ Materials Science and Engineering Program \\ ${ }^{3}$ Department of Mechanical Engineering \\ ${ }^{4}$ Stem Cell Center \\ University of California - Riverside \\ Riverside, CA, 92521, USA \\ *Email: mprao@engr.ucr.edu
}

\author{
R. Starr, ${ }^{1}$ X. Yang, ${ }^{1}$ Dr. S. J. Forman, ${ }^{1,2}$ Dr. C. E. Brown ${ }^{1,2}$ \\ ${ }^{1}$ Department of Cancer Immunotherapy and Tumor Immunology \\ ${ }^{2}$ Department of Hematology and Hematopoietic Cell Transplantation \\ City of Hope Beckman Research Institute and Medical Center \\ Duarte, CA, 91010, USA \\ Dr. C. B. Ballas ${ }^{1}$ \\ ${ }^{1}$ Division of Hematology/Oncology \\ Indiana University School of Medicine \\ Indianapolis, IN, 46202, USA
}




\section{MATERIALS AND METHODS}

Device Fabrication. The DMP devices were fabricated using $100 \mathrm{~mm}$ diameter silicon-oninsulator substrates with $20 \mu \mathrm{m}$ device, $2 \mu \mathrm{m}$ buried $\mathrm{SiO}_{2}(\mathrm{BOX})$, and $500 \mu \mathrm{m}$ handle layers (Ultrasil Corporation). Front and backside $\mathrm{SiO}_{2}$ etch masks with $1 \mu \mathrm{m} \& 2 \mu \mathrm{m}$ thicknesses, respectively, were first deposited using a combination of wet oxidation (CVD Equipment Corp; $7 / 4 \mathrm{sccm} \mathrm{H} / \mathrm{O}_{2} \& 1000^{\circ} \mathrm{C}$ ) and plasma enhanced chemical vapor deposition (Plasmatherm 790, Unaxis: $400 / 900 \mathrm{sccm} 2 \% \mathrm{SiH}_{4} / \mathrm{N}_{2} \mathrm{O}, 900 \mathrm{mT}$, \& $\left.25 \mathrm{~W}\right)$. The frontside Aspiration Via oxide mask was then patterned by projection lithography (GCA 6300, RTC) and fluorine-based reactive ion etching (RIE) (Multiplex RIE, STS: 30/20 sccm $\mathrm{CHF}_{3} / \mathrm{CF}_{4}, 100 \mathrm{mT}$, \& $300 \mathrm{~W}$ ). This was followed by Backside Aspiration Port oxide mask patterning using contact lithography (MA-6, Suss Microtec) and fluorine-based RIE. The Backside Aspiration Port was then defined using Si deep reactive ion etching (DRIE) (MESC ICP, STS: Etch cycle - 130/13 sccm SF $6 / \mathrm{O}_{2}, 37 \mathrm{mT}$, 700/20 W source/platen, \& $14 \mathrm{~s}$; Passivation cycle $-85 \mathrm{sccm} \mathrm{C}_{4} \mathrm{~F}_{8}, 24 \mathrm{mT}, 600 / 0 \mathrm{~W}$ source/platen, \& $7 \mathrm{~s}$ ). Afterwards, the Capture Wells and Penetrators were simultaneously defined using frontside isotropic Si RIE (MESC ICP: 95/13 sccm SF $/ \mathrm{O}_{2}, 12 \mathrm{mT}$, \& 500/20 W source/platen). The Aspiration Vias were then defined by frontside Si DRIE using the Aspiration Via oxide mask as a shadow-mask (MESC ICP: Etch cycle - 130/13 sccm SF $6 / \mathrm{O}_{2}, 24 \mathrm{mT}, 600 / 17 \mathrm{~W}$ source/platen, \& 7 s; Passivation cycle - 85 sccm $\mathrm{C}_{4} \mathrm{~F}_{8}, 14$ mT, 600/0 W source/platen, \& 5 s). Finally, the residual frontside oxide mask was removed using fluorine-based RIE, followed by Penetrator tip refinement by chlorine-based RIE (E640, Panasonic Factory Solutions: $10 \mathrm{sccm} \mathrm{Cl}, 1.2 \mathrm{~Pa}, \& 400 / 12 \mathrm{~W}$ source/platen) and fluorine-based RIE (Multiplex RIE: 40/50 sccm O $/ \mathrm{CF}_{4}, 100 \mathrm{mT}$, \& $300 \mathrm{~W}$ ), and lastly, BOX layer removal by backside fluorine-based RIE (Multiplex RIE: 30/20 sccm $\mathrm{CHF}_{3} / \mathrm{CF}_{4}, 100 \mathrm{mT}$, \& $300 \mathrm{~W}$ ). Scanning electron 
microscopy was used throughout for fabrication process characterization and device feature verification (Leo Supra 55, Zeiss).

Device Packaging and Experimental Apparatus. The device package was fabricated from polycarbonate and designed for placement on the stage of an upright fluorescence microscope (BX50, Olympus). The microscope was equipped with a CCD camera (Retiga EXi, Q Imaging) and high-intensity lamp (Sola Light Engine, Lumencor), which enabled real-time visualization during device operation. A programmable syringe pump (PhD 2000, Harvard Apparatus) was used for bidirectional actuation of the aspiration circuit.

Cell Culture. The Jurkat and K-562 cells (ATCC) were each cultured at $37^{\circ} \mathrm{C}$ and $5 \% \mathrm{CO}_{2}$ in RPMI medium (1640, Lonza), with 10\% fetal bovine serum (FBS, Hyclone Labs). The media was refreshed every $48 \mathrm{~h}$, and the cultures were passaged at population densities of $10^{6} \mathrm{cells} / \mathrm{mL}$ (MUSE, EMD Millipore). Primary T cell culture was derived from human donor $\mathrm{Tn} / \mathrm{mem}$ population and maintained under $37^{\circ} \mathrm{C}$ and $5 \% \mathrm{CO}_{2}$ in X-Vivo15 media (BioWhittaker) containing $10 \%$ fetal calf serum (HyClone, GE Healthcare), supplemented with $50 \mathrm{U} / \mathrm{mL}$ recombinant human (rh) IL-2, and $0.5 \mathrm{ng} / \mathrm{mL}$ rhlL-15. ${ }^{1}$ Cells were stimulated with Dynabeads Human T Expander CD3/CD28 (Invitrogen) for $7 \mathrm{~d}$ and expanded out to days 18 - 25 for experiments.

Capture Optimization. Prior to their introduction into the DMP device, the Jurkats were stained with $10 \mu \mathrm{M}$ Calcein Blue AM (CBAM, Life Technologies) in phosphate-buffered saline (PBS, Life Technologies) for $30 \mathrm{~min}$. The cells were then centrifuged and resuspended in fresh PBS with 4\% glycerol (to enhance cell settling during the capture process). The device was infused with fresh PBS in an amount sufficient to partially fill the reservoir, and the CBAM-stained cells were pipetted into the reservoir, followed by actuation of the aspiration circuit using the syringe pump. Pipetting was then used to wash away uncaptured cells from the device, and subsequently 
remove them from the reservoir. Finally, the fluorescence microscope was used to build a photomosaic of the captured cells across the entirety of the device array. Capture Site occupancy (i.e., \% of occupied Capture Sites) was determined using image analysis software (Image J, NIH), coupled with a machine learning segmentation plugin (WEKA, University of Waikato, New Zealand). The analysis parameters were defined to exclude both cellular debris and cellular aggregates.

Puncture Optimization. The device was first infused with $0.1 \mu \mathrm{g} / \mathrm{mL}$ propidium iodide (PI, Sigma-Aldrich) in PBS. CBAM-stained Jurkats in $4 \%$ glycerol/PBS were then pipetted into the reservoir, captured at $30 \mu \mathrm{L} / \mathrm{min}$, and punctured at flow rates ranging from $20 \mu \mathrm{L} / \mathrm{min}$ to $60 \mu \mathrm{L} / \mathrm{min}$. Uncaptured cells were then removed from the device by pipette-based washing, followed by reversal of the aspiration flow to release the captured cells. The released cells were then collected from the reservoir, and co-incubated with $0.1 \mu \mathrm{g} / \mathrm{mL}$ PI and $1 \mu \mathrm{M}$ CellTracker Green CMFDA Dye (CTG, Life Technologies) for 30 min. Finally, the cells were centrifuged, resuspended in FACS stain solution (Gibco), and assayed using flow cytometry (MACSQuant Analyzer, Miltenyi Biotec). Subsequent analysis of the flow cytometry data was performed using a commercial software package (FCS Express 6, De Novo Software). Using a cascading gating scheme based on control data reported in Fig. S1 in the Supporting Information, cellular viability was defined as the percentage of viable intact cells, relative to the population of intact cells recovered from the device (i.e., Gate 2/Gate $1 \times 100 \%$ ). Delivery efficiency was defined as the percentage of viable intact cells with exogenous cargo delivered, relative to the population of viable intact cells (i.e., Gate $3 /$ Gate $2 \times 100 \%$ ). Finally, delivery yield was defined as the percentage of viable intact cells with exogenous cargo delivered, relative to the population of intact cells (i.e., Gate 3/Gate $1 \mathrm{x}$ 100 , which is equivalent to the product of cellular viability and delivery efficiency). 
DMP Validation. The device was first infused with $20 \mu \mathrm{g} / \mathrm{mL}$ GFP plasmid in PBS. Jurkat, K562, or primary human T cells previously stained using $4 \mu \mathrm{M}$ CellTrace Calcein Red-Orange AM (ThermoFisher), and resuspended in $4 \%$ glycerol/PBS, were then introduced into the device, captured at $30 \mu \mathrm{L} / \mathrm{min}$, and punctured at $40 \mu \mathrm{L} / \mathrm{min}$. Uncaptured cells were then removed from the device by pipette-based washing, followed by reversal of the aspiration flow to release the captured cells. The released cells were then collected from the reservoir, and incubated in GFP plasmid/PBS for 30 min. Afterwards, they were centrifuged, resuspended in fresh RPMI with $0.5 \%$ Penicillin-Streptomycin, and incubated in a humidified $37^{\circ} \mathrm{C} / 5 \% \mathrm{CO}_{2}$ incubator for $12 \mathrm{~h}$. Finally, the cells were centrifuged, stained with $8 \mu \mathrm{M}$ CBAM in PBS for $30 \mathrm{~min}$, followed by centrifugation and resuspension in FACS stain for flow cytometry. Cellular viability, transfection efficiency, and transfection yield were defined in a similar manner as the puncture optimization studies (with gating based on control data reported in Fig. S2).

BEP Benchmarking. The benchmarking studies were performed using a commercial BEP instrument (Nucleofector 1, Lonza), with manufacturer-recommended protocols and plasmid concentrations for Jurkats (i.e., Cell Line Nucleofector Kit V, Program X-01, and $20 \mu \mathrm{g} / \mathrm{mL}$ GFP plasmid), K-562 cells (i.e., Cell Line Nucleofector Kit V, Program T-03, and 20 g/mL GFP plasmid), and primary human T cells (i.e., Human T Cell Nucleofector Kit, Program U-14, and $20 \mu \mathrm{g} / \mathrm{mL}$ GFP plasmid). Afterwards, the cells were collected from the instrument, resuspended in fresh media with $0.5 \%$ Penicillin-Streptomycin, and incubated in a humidified $37^{\circ} \mathrm{C} / 5 \% \mathrm{CO}_{2}$ incubator for $12 \mathrm{~h}$. Finally, the cells were centrifuged, stained with $0.80 \mu \mathrm{M}$ 4,6-diamidino-2-phenylindole (DAPI, ThermoFisher) in PBS for 30 min, followed by centrifugation and resuspension in FACS stain for flow cytometry. Cellular viability, transfection efficiency, and transfection yield were defined in 
the same manner as the DMP validation studies (with gating based on control data reported in

Figs. S3, S4, \& S5 for Jurkat, K-562, \& primary human T cells, respectively).

Statistical Analyses. All cell studies were repeated in triplicate and statistical analyses

were performed using the student's t-test with two-tailed distribution and two-sample unequal

variance (Excel, Microsoft).

\section{REFERENCES}

1. Brown, C. E.; Aguilar, B.; Starr, R.; Yang, X.; Chang, W.-C.; Weng, L.; Chang, B.; Sarkissian, A.; Brito, A.; Sanchez, J. F.; et al., Optimization of IL13Ra2-Targeted Chimeric Antigen Receptor T Cells for Improved Anti-Tumor Efficacy against Glioblastoma. Molecular Therapy 2018, 26 (1), 31-44.

\section{a)}

b)
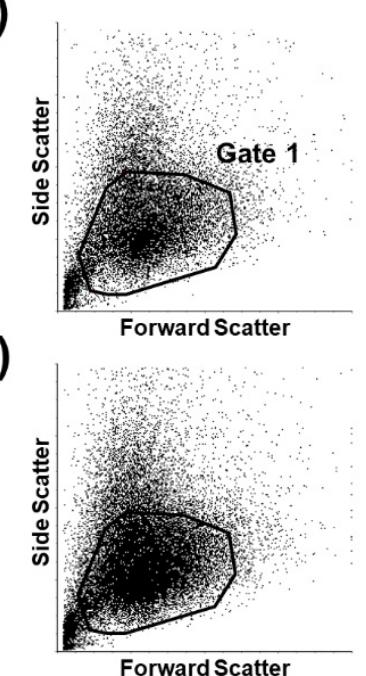

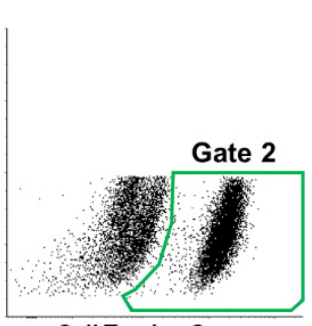

Cell Tracker Green

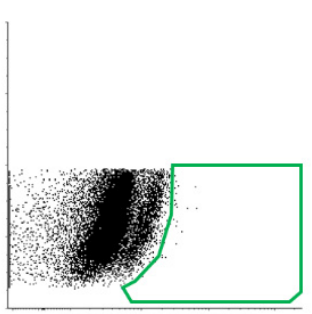

Cell Tracker Green

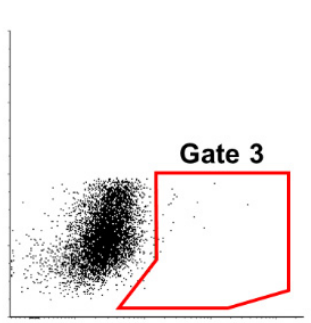

Propidium lodide

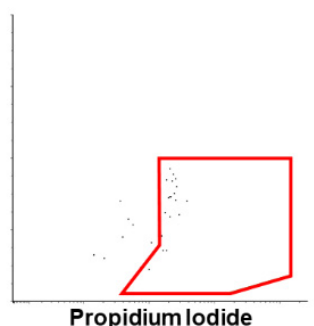

Figure S1. Representative flow cytometry control data used to establish the cascading gating scheme for the puncture optimization study (i.e., Fig. 3), with Jurkats serving as a model suspension cell line, propidium iodide (PI) serving as a model, membraneimpermeable, small-molecule exogenous cargo, and Cell Tracker Green (CTG) serving as a post-DMP cellular viability marker. (a) Non-porated cell control. The cells were subjected to the same pre- and post-DMP protocols used for the puncture optimization samples. However, rather than running the cells through the DMP device, they were instead incubated in PBS/glycerol for 7 min to simulate total time in the device. Gates 1, 2, and 3 encompass the population of intact cells (i.e., events with size and granularity consistent with intact cells), viable intact cells (i.e., CTG+), and viable intact cells with passive uptake of the exogenous cargo (i.e., both $\mathrm{CTG}+\& \mathrm{PI}+$ ), respectively. The high proportion of cells in Gate 2, and low proportion in Gate 3, indicated good viability and minimal passive uptake of PI, respectively, in the non-porated cells. (b) Autofluorescence control. The cells were not subjected to any staining, nor poration. Instead, they were simply incubated in PBS/glycerol for 7 min to simulate total time in the DMP device, followed immediately by incubation in PBS for another $30 \mathrm{~min}$ to simulate the total time of the post-DMP staining used for the puncture optimization samples. Gates 1,2 , and 3 encompass the population of intact cells, intact CTG+ cells, and intact CTG+ \& $\mathrm{PI}+$ cells, respectively. The low proportions of cells in Gates 2 and 3 indicated that there was negligible autofluorescence in these spectral ranges. 
a)

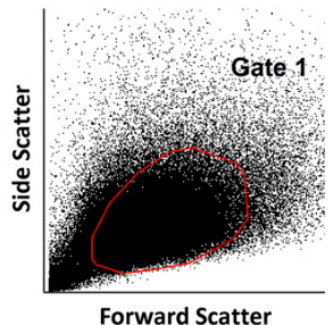

b)

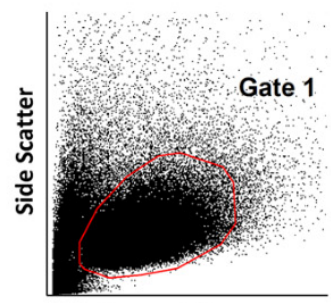

Forward Scatter

c)

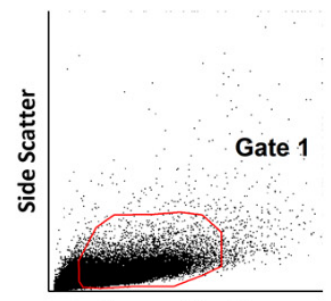

Forward Scatter

d)

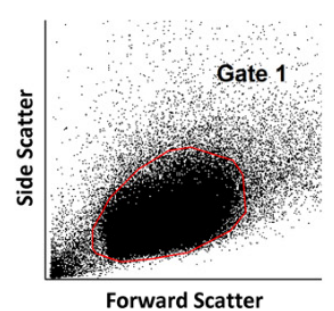

e)

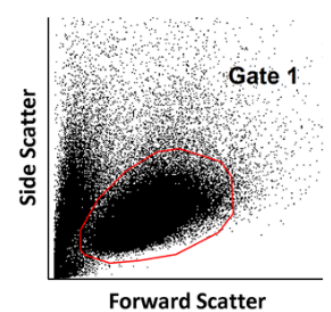

f)

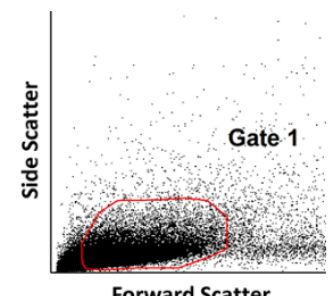

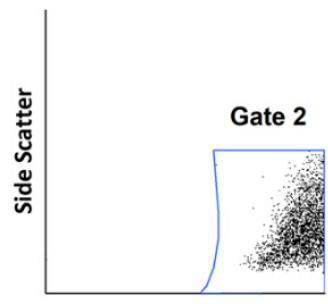

Calcein Blue AM

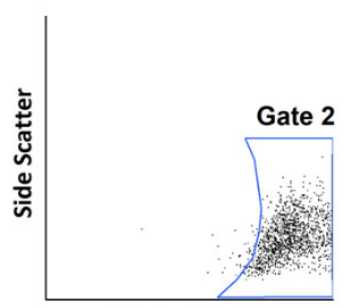

Calcein Blue AM

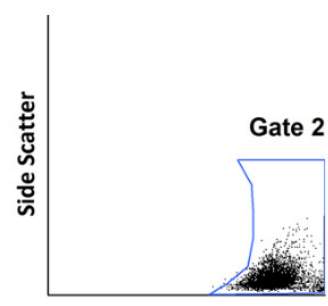

Calcein Blue AM

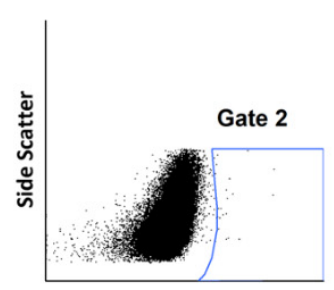

Calcein Blue AM

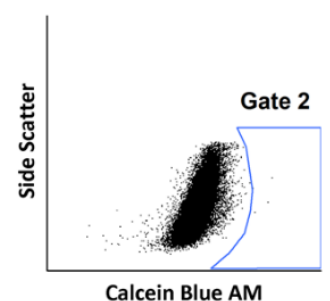

Calcein Blue AM

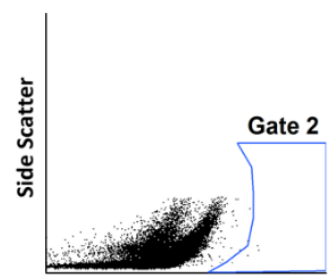

Calcein Blue AM

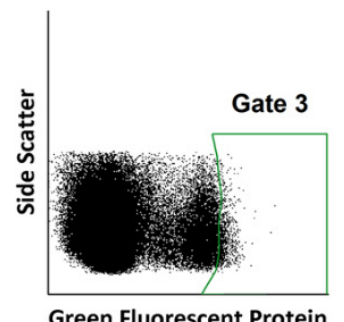

Green Fluorescent Protein

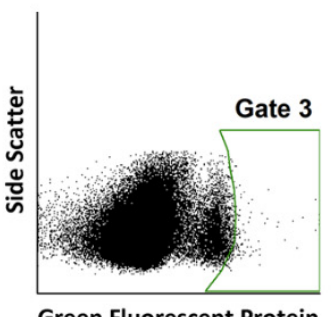

Green Fluorescent Protein

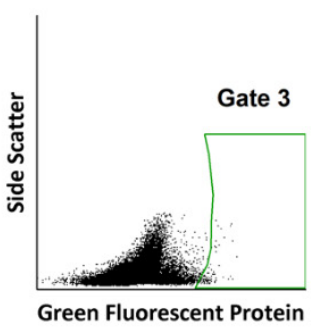

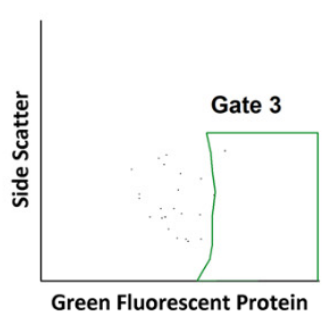

Green Fluorescent Protein
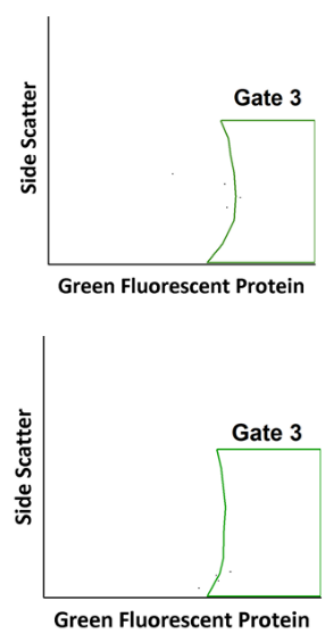
Figure S2. Representative control data used to establish the cascading gating scheme for the DMP validation study (i.e., Fig. 4), using Jurkat, K-562, and primary human T cells, with GFP plasmid serving as a model genetic construct cargo, and Calcein Blue AM (CBAM) serving as a post-incubation cellular viability marker. ( $a, b, c)$ Non-porated cell controls for Jurkat, K-562, and primary human T cells, respectively. The cells were subjected to the same pre- and post-DMP protocols used for the DMP validation samples. However, rather than running the cells through the DMP device, they were instead incubated in PBS/glycerol for 7 min to simulate total time in the device. Gates 1, 2, and 3 encompass the population of intact cells, viable intact cells (i.e., CBAM+), and viable intact cells with passive uptake and expression of the plasmid cargo (i.e., both CBAM+ \& GFP+), respectively. The high proportion of cells in Gate 2, and low proportion in Gate 3, indicated good viability and minimal passive uptake and expression of the plasmid, respectively, in the non-porated cells. (d, e, f) Autofluorescence controls for Jurkat, K-562, and primary human T cells, respectively. The cells were not subjected to any staining, plasmid exposure, or poration. Instead, they were simply incubated in PBS/glycerol for 7 min to simulate total time in the DMP device, followed immediately by incubation in PBS for another 30 min to simulate the total time of the post-DMP plasmid incubation, and finally, followed by the same $12 \mathrm{~h}$ incubation protocol used for the DMP validation samples. Gates 1,2 , and 3 encompass the population of intact cells, intact CBAM+ cells, and intact CBAM+ \& GFP+ cells, respectively. The low proportions of cells in Gates 2 and 3 indicated that there was negligible autofluorescence in these spectral ranges. 
a)

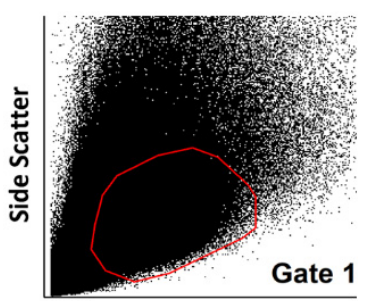

b)

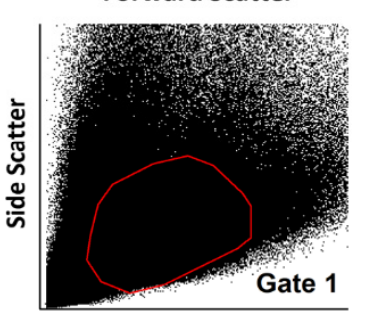

c)

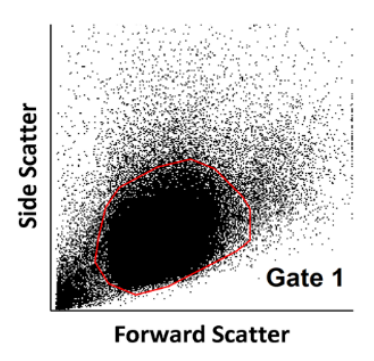

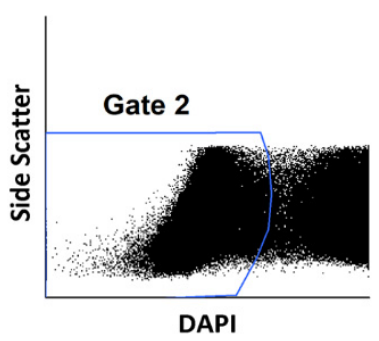
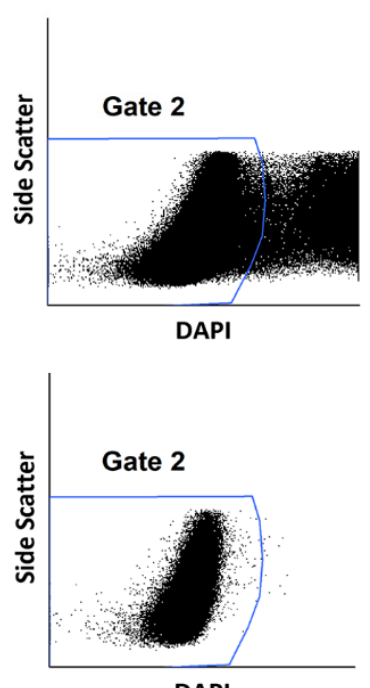

Gate 3

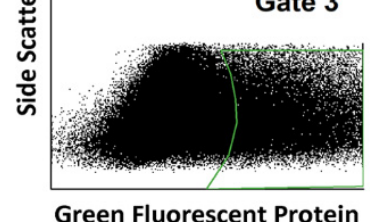

Green Fluorescent Protein
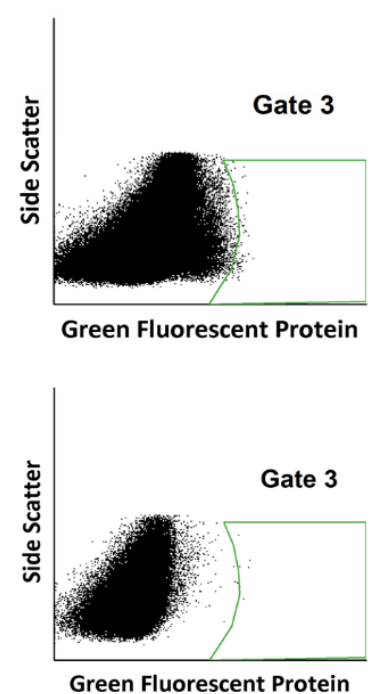

Figure S3. Representative Jurkat BEP benchmarking data and controls for the DMP validation study (i.e., Fig. 4), with GFP plasmid serving as the cargo, and DAPI serving as a post-incubation cellular viability marker. (a) Representative flow cytometry data for BEP cells. Gates 1, 2, and 3 encompass the population of intact cells, viable intact cells (i.e., DAPI-), and viable intact cells with delivery and expression of the plasmid cargo (i.e., both DAPI- \& GFP+), respectively. (b) Non-porated cell control. The cells were subjected to the same pre- and post-BEP protocols used for the BEP samples. However, rather than running the cells through the BEP instrument, they were instead incubated in fresh media with GFP for $7 \mathrm{~min}$ to simulate total time in the instrument. Gates 1 , 2 , and 3 encompass the population of intact cells, viable intact cells (i.e., DAPI-), and viable intact cells with passive uptake and expression of the plasmid cargo (i.e., both DAPI- \& GFP+), respectively. The high proportion of cells in Gate 2, and low proportion in Gate 3, indicated good viability and minimal passive uptake and expression of the plasmid cargo, respectively, in the nonporated cells. (c) Autofluorescence control. The cells were not subjected to any staining, plasmid exposure, or poration. Instead, they were simply incubated in fresh media for $1 \mathrm{~min}$ to simulate total time in the BEP instrument, followed by the same $12 \mathrm{~h}$ incubation protocol used for the BEP samples. Gates 1, 2, and 3 encompass the population of intact cells, intact DAPI- cells, and intact DAPI- \& GFP+ cells, respectively. The low proportion of cells outside of Gate 2, and the low proportion within Gate 3 indicated that there was negligible autofluorescence in these spectral ranges. 


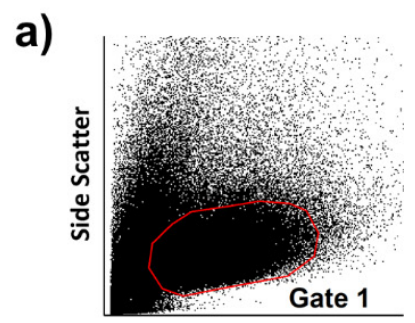

b)

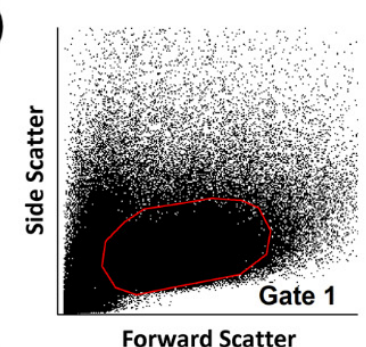

c)

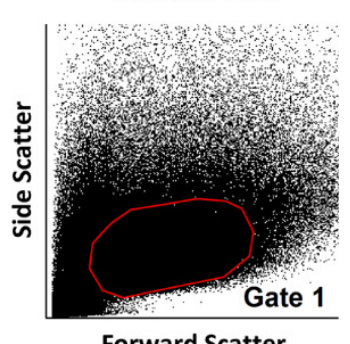

Forward Scatter

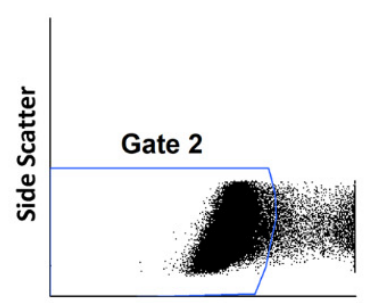

DAPI

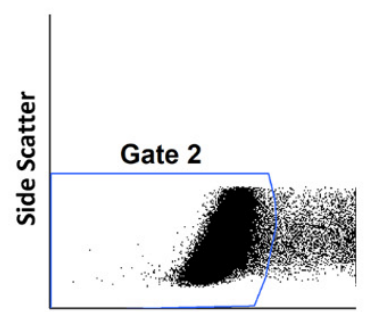

DAPI

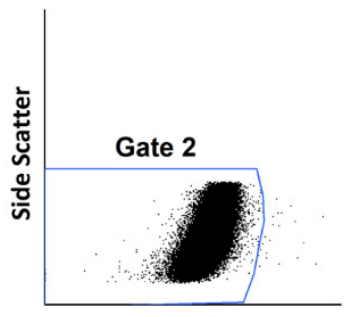

DAPI

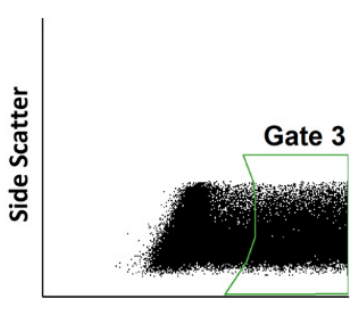

Green Fluorescent Protein

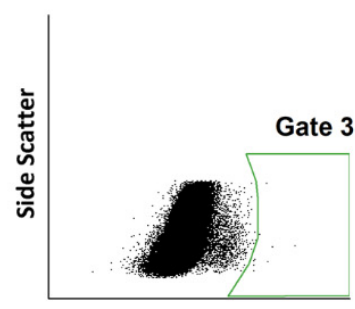

Green Fluorescent Protein

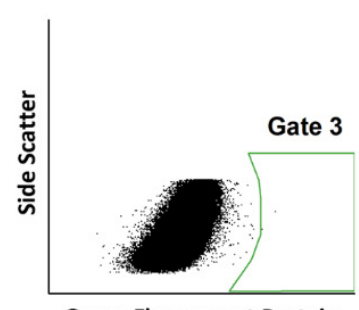

Green Fluorescent Protein

Figure S4. Representative K-562 BEP benchmarking data and controls for the DMP validation study (i.e., Fig. 4), with GFP plasmid serving as the cargo, and DAPI serving as a post-incubation cellular viability marker. (a) Representative flow cytometry data for BEP cells. Gates 1, 2, and 3 encompass the population of intact cells, viable intact cells (i.e., DAPI-), and viable intact cells with delivery and expression of the plasmid cargo (i.e., both DAPI- \& GFP+), respectively. (b) Non-porated cell control. The cells were subjected to the same pre- and post-BEP protocols used for the BEP samples. However, rather than running the cells through the $B E P$ instrument, they were instead incubated in fresh media with GFP for 7 min to simulate total time in the instrument. Gates 1 , 2 , and 3 encompass the population of intact cells, viable intact cells (i.e., DAPI-), and viable intact cells with passive uptake and expression of the plasmid cargo (i.e., both DAPI- \& GFP+), respectively. The high proportion of cells in Gate 2, and low proportion in Gate 3, indicated good viability and minimal passive uptake and expression of the plasmid cargo, respectively, in the nonporated cells. (c) Autofluorescence control. The cells were not subjected to any staining, plasmid exposure, or poration. Instead, they were simply incubated in fresh media for $1 \mathrm{~min}$ to simulate total time in the BEP instrument, followed by the same $12 \mathrm{~h}$ incubation protocol used for the BEP samples. Gates 1, 2, and 3 encompass the population of intact cells, intact DAPI- cells, and intact DAPI- \& GFP+ cells, respectively. The low proportion of cells outside of Gate 2, and the low proportion within Gate 3 indicated that there was negligible autofluorescence in these spectral ranges. 


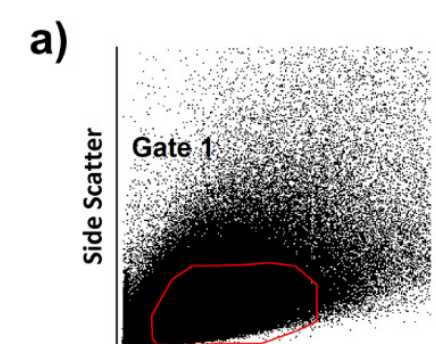

Forward Scatter

b)

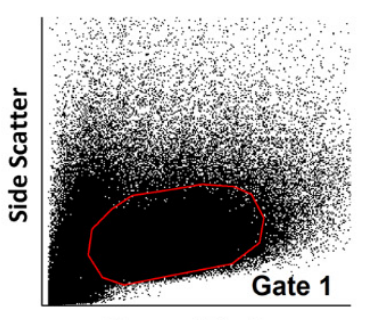

c)

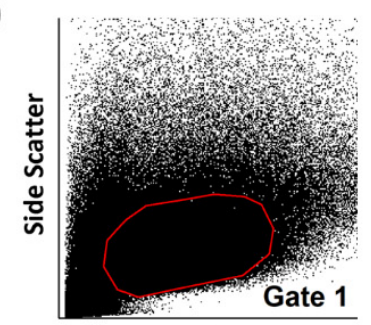

Forward Scatter

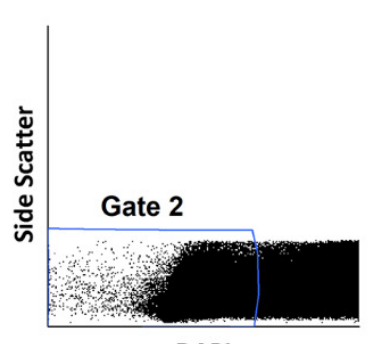

DAPI

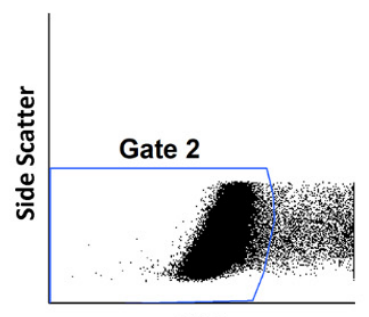

DAPI

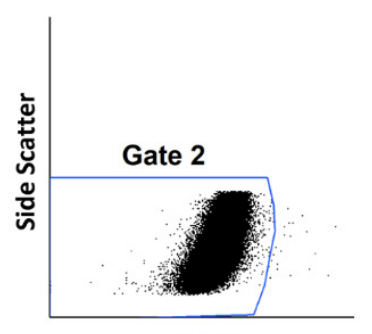

DAPI

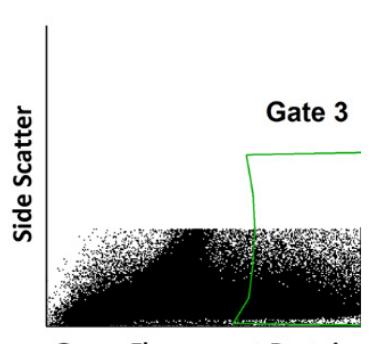

Green Fluorescent Protein

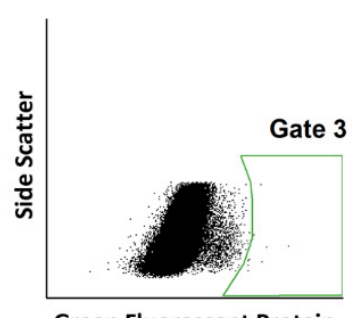

Green Fluorescent Protein

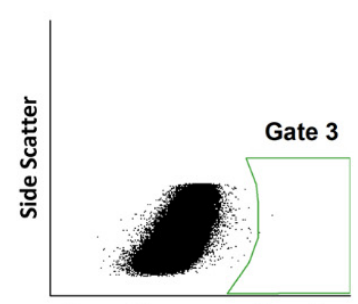

Green Fluorescent Protein

Figure S5. Representative primary human T cell BEP benchmarking data and controls for the DMP validation study (i.e., Fig. 4), with GFP plasmid serving as the cargo, and DAPI serving as a post-incubation cellular viability marker. (a) Representative flow cytometry data for BEP cells. Gates 1, 2, and 3 encompass the population of intact cells, viable intact cells (i.e., DAPI-), and viable intact cells with delivery and expression of the plasmid cargo (i.e., both DAPI- \& GFP+), respectively. (b) Non-porated cell control. The cells were subjected to the same pre- and post-BEP protocols used for the BEP samples. However, rather than running the cells through the BEP instrument, they were instead incubated in fresh media with GFP for 7 min to simulate total time in the instrument. Gates 1, 2, and 3 encompass the population of intact cells, viable intact cells (i.e., DAPI-), and viable intact cells with passive uptake and expression of the plasmid cargo (i.e., both DAPI- \& GFP+), respectively. The high proportion of cells in Gate 2 , and low proportion in Gate 3, indicated good viability and minimal passive uptake and expression of the plasmid cargo, respectively, in the non-porated cells. (c) Autofluorescence control. The cells were not subjected to any staining, plasmid exposure, or poration. Instead, they were simply incubated in fresh media for $1 \mathrm{~min}$ to simulate total time in the BEP instrument, followed by the same $12 \mathrm{~h}$ incubation protocol used for the BEP samples. Gates 1, 2, and 3 encompass the population of intact cells, intact DAPI- cells, and intact DAPI- \& GFP+ cells, respectively. The low proportion of cells outside of Gate 2, and the low proportion within Gate 3 indicated that there was negligible autofluorescence in these spectral ranges. 